\title{
Effects of Pattern Complexity on Information Integration: Evidence from Eye Movements
}

\author{
Yanju Ren ${ }^{1,2}$, Yuming Xuan ${ }^{1}$, and Xiaolan $\mathrm{Fu}^{1, *}$ \\ ${ }^{1}$ State Key Laboratory of Brain and Cognitive Science, Institute of Psychology, \\ Chinese Academy of Sciences, \\ Beijing 100101, China \\ ${ }^{2}$ Graduate School of the Chinese Academy of Sciences, \\ Beijing, 100049, China \\ \{renyj, xuanym, fuxl\}@psych.ac.cn
}

\begin{abstract}
The present study employed empty cell localization paradigm and eye-tracking method to investigate the effects of memorized stimuli complexity on information integration between in visual short-term memory (VSTM) and visual perception. Two arrays of dots were displayed in sequence within a grid. Between the two arrays, one cell was always empty, and the participants' task was to specify the location of this "missing dot" It was found that the accuracy decreased as dot pattern of array 1 increased in complexity, especially under long ISI condition. The analysis of eye movement behavior, especially fixation location, demonstrated that participants were more likely to try to remember the location of the empty cells of array 1 other than locations of dots. From aspect of eye movement, these results offered the first evidence supporting convertand-compare hypothesis.
\end{abstract}

Keywords: information integration, visual short-term memory, visual perception, eye movement.

\section{Introduction}

Visual short-term memory (VSTM) allows the contents of visual perception to be retained momentarily to create temporal continuity in a constantly changing visual environment [12] [18], yet it has severe capacity limits. Only four objects or six spatial locations can be retained in VSTM [14] [17]. Research effort in the past decade has focused primarily on the representation of a single visual display. Yet, in many everyday activities, visual information not only occupies space but also evolves over time. In order to keep coherent visual environment, we need to integrate the information retained in the VSTM and visual percept. Driving requires the constant updating of information gathered from many instances. When interacting with computer we need to integrate information from different windows.

Recently, empty cell localization paradigm, used to investigate perceptual integration [5] [6] [7] [9] was also used to examine the integration between VSTM

\footnotetext{
* Corresponding author.
} 
and visual perception [1] [2] [3] [4] [10] [13]. In this paradigm, two arrays of dots were displayed in sequence within a visible or virtual grid. The first array filled approximately half of the cells in the grid. Array 2 filled all but one of the cells not filled in Array 1. Between the two arrays, one cell was always empty, and the task was to specify the location of this "missing dot".

To date, two hypotheses were proposed for the mechanism of integration between VSTM and visual perception. One was image-percept integration hypothesis, which assumed visual perception could be integrated with the contents of VSTM [1] [2] [3] [4]. When an observer generated a representation of array 1 in memory, a subsequently perceived array 2 could be directly incorporated into the existing representation. The other was convert-and-compare hypothesis, which assumed the first array might be used to visually mark grid positions as locations that cannot constitute the correct answer [21] [22]. As a result, these positions might be inhibited, and attention directed to the positions that Array 1 left empty, enhancing the detection of the space left unfilled by the second array [10] [13].

The convert-and-compare hypothesis postulated that VSTM had a small capacity of about six spatial locations, and this hypothesis was consistent with limited capacity of VSTM [14] [17]. However, the image-percept integration hypothesis postulated that VSTM has a higher capacity of about 14-20 locations, and that it supports integration across sequential arrays [2] [4]. Given this hypothesis was correct, then what accounted for this apparently high-capacity memory, and could these data be reconciled with current views of limited-capacity VSTM? Hollingworth et al (2005) pointed out that it is likely that long-ISI performance was supported by VSTM, and VSTM grouped individual dots into a larger scale object or objects [10]. They tested this figural grouping hypothesis and found that pattern complexity had no effect on empty cell localization at 0 -msec ISI, but there was a large simple pattern advantage at long ISIs.

In the present experiment, we continued to use Hollingworth et al's method to test further this figural grouping hypothesis [10]. More importantly, we monitored participants' eye movement behavior during integration in order to determine the locus of overt attention and to uncover the mechanism underlying the integration process. If the image-percept integration hypothesis is correct, participants should pay more attention to locations occupied by dots when viewing array 1; On the contrary, if the convert-and-compare hypothesis is correct, participants should pay more attention to empty cells instead.

\section{Method}

\subsection{Participants}

Twelve undergraduates (6 male and 6 female, their average ages were 21) from the China Agricultural University participated in the experiment. All participants reported normal or corrected-to-normal vision. After the experiment, they were paid 25 RMB.

\subsection{Stimuli}

The stimuli were similar to those used in Hollingworth et al (2005, Experiment 2) [10]. Two dot arrays were displayed on each trial within a 4 x 4 grid. The Array 1 
stimuli were drawn from three sets: a simple figure set, a medium figure set and a complex figure set. Simple, medium and complex figures were generated based on complexity ratings compiled by Ichikawa [11]. Each stimulus collected by Ichikawa was a 4 x 4 grid with eight of the cells filled by dots. A total of 140 different patterns were rated by participants, and the stimuli were ordered from least complex (item 1) to most complex (item 140). The set of 32 simple, medium, and complex figure stimuli for the experiment were items 1-32, 55-86, and 109-140 from Ichikawa respectively. Sample stimuli from the simple, medium and complex figure set were showed in Fig. 1. The Array 2 stimuli were constructed by randomly filling seven of the eight cells not filled in Array 1. As a result, the position of the empty cell was also randomly determined. The 4 x 4 grid was composed of light-blue lines superimposed over the background (was light gray and subtended $36^{\circ}$ x $27^{\circ}$ of visual angle). Dots were presented in black. The entire grid subtended $20^{\circ}$ of visual angle (both horizontally and vertically). Each cell in the grid subtended $5^{\circ}$. The diameter of each dot was $4^{\circ}$.
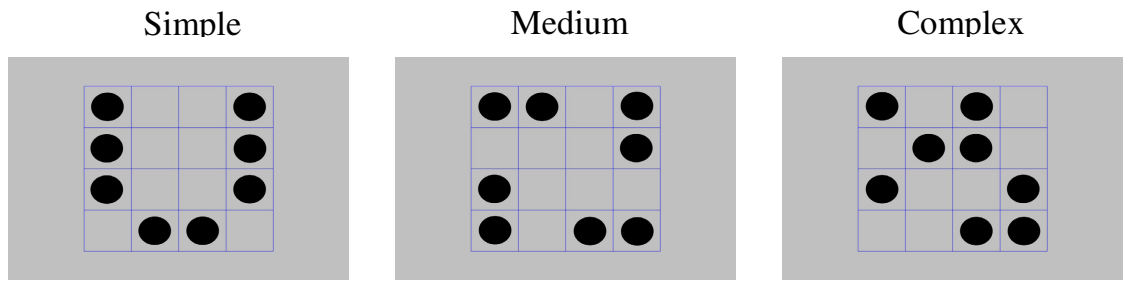

Fig. 1. Sample Array 1 stimulus from the simple pattern set (left), from the medium pattern set (middle) and from the complex pattern set (right) in the present experiment

\subsection{Apparatus}

The stimuli were presented at a resolution of 1024 by 768 pixels on a 19-inch video monitor at a refresh rate of $85 \mathrm{~Hz}$. The presentation of stimuli and collection of responses was controlled by Experiment Builder software running on a Pentium IV PC. Viewing distance was maintained at about $60 \mathrm{~cm}$. Eye position was sampled at a rate of $500 \mathrm{~Hz}$ (every $2 \mathrm{~ms}$ ) with an EyeLink II eyetracker (SR Research Ltd. in Canada) with a resolution of $0.01^{\circ}$ in pupil only mode. The eyetracker and display monitor were interfaced with a computer that controlled the experiment. This system used video-based infrared oculography to measure eye and head position.

\subsection{Design and Procedure}

In general, the design and procedure were very similar to that used by Hollingworth et al (2005) [10]. On each trial, two dot arrays (eight and seven dots, respectively) were presented sequentially within an enclosed square grid separated by a variable ISI. There were three blocks of trials totally, with each level of pattern complexity (simple, medium and complex) comprising a block. On any given trial, one cell within the grid was never filled and the participants were instructed to identify the position of the empty cell. 
The procedure was illustrated in Fig. 2 and each trial consisted of the following events. Firstly, the black point was presented in the center of the screen. When ready to begin, participants fixated the black point and pressed '5' on the Eye Link Button Controller to start the trial. There was the empty grid (superimposed over the gray background) which was presented for $500 \mathrm{~ms}$ delay before presentation of Array1. Array 1 was then presented within the grid for $35 \mathrm{~ms}$ (about three refresh cycles at 85 $\mathrm{Hz}$ ). The variable ISI between the offset of Array 1 and the onset of Array 2 was 100, 750,1500 , or $2500 \mathrm{~ms}$. During the ISI, the blank grid was displayed. Following the ISI, Array 2 was presented within the grid for $35 \mathrm{~ms}$. Array 2 was followed by the grid with the 16 numbers from '1' to '16' presented in the center of each cell. Participants identified the location of the empty cell by speaking out the number standing for the cell. Then participants pressed '5' on the Eye Link Button Controller to start the next trial. Participants were asked to respond as accurately as possible and that they were under no speed stress.

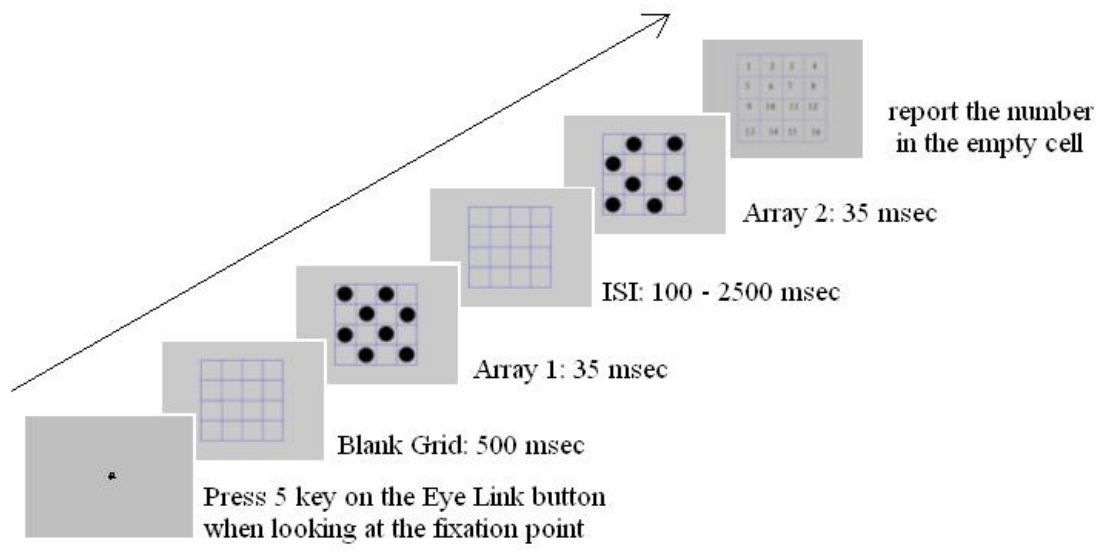

Fig. 2. Sequence of events in a trial of the experiment. The participants pressed 5 key on the Eye Link button to begin the trial, followed by the events illustrated in the figure. When the final blank grid with number appeared, the participants should speak out the number in the empty cell.

Participants were tested individually. Before tested, each participant was given a written description of the experiment along with a set of instructions. The experiment would get started after each participant understood the instructions.

The experiment consisted of two sessions: practice session and formal experiment session. The procedure of practice session was compiled by E-prime 1.1 and was similar to the formal experiment session except that there was no the black point screen and participant moved mouse to click the empty cell. Participants first completed a practice session of 16 trials, four in each of 4 ISI conditions, randomly intermixed. Feedback was provided in the practice session with the $1000 \mathrm{~ms}$ gray screen between trials either contained the word "correct" or "incorrect". During the 
practice session, eye movements of participants were not recorded. The experimental session, consisted of three blocks (simple pattern, medium pattern and complex pattern) of trials. Each block contained 128 trials and stimuli (32)-ISI (4) assignments were rotated within blocks by Latin square. The blocks' order was counterbalanced by Latin square between participants. Participants began each trial by fixating a center point. The position of the dominant eye was tracked, though viewing was binocular. At the beginning of each block, the eyetracker was calibrated and validated. Once calibration and validation were completed, the experimental trials began.

The eye tracker monitored eye position during the presentation of Array 1, ISI, and the presentation of Array 2. The participants began each trial by looking at the center of the grid but were free to move their eye without constraints during the trial. Eye position was scored in terms of the grid space that was fixated. Saccades were operationally defined as a change in fixation that exceeded $0.15^{\circ}$ of visual angle accompanied by a velocity exceeding $30 \% \mathrm{sec}$ or an acceleration exceeding $8000 \% \mathrm{sec}^{2}$ that was maintained for a minimum of $2 \mathrm{~ms}$. The saccade was considered to be terminated when there criteria were no longer met.

The participants were not informed of the pattern complexity manipulation. Feedback was not provided in the experimental session. There were two-minute break between practice session and experimental session and ten-minute breaks between successive blocks. The entire experiment lasted approximately $90 \mathrm{~min}$.

\section{Results}

The results were reported in two parts. First, general accuracy and error rates for different error types in the information integration task were reported. Second, various aspects of eye movement behavior were examined. Variables of interest included fixation number, fixation location, fixation duration and pupil size.

\subsection{Integration Task Accuracy and Error Rate}

A response was classified as correct, an Array 1 error (erroneously selecting a position occupied by the first array), or an Array 2 error (erroneously selecting a position occupied by the second array) and was measured in terms of the percentage of trials on which they occurred. The accuracy, percentage of Array 1 error and percentage of Array 2 error in the empty cell localization task was examined as a function of pattern complexity of Array 1 and ISI.

Accuracy. There were a reliable main effect of complexity $[F(2,22)=70.159, p<$ $.001]$, a reliable main effect of ISI $[F(3,33)=52.565, p<.001]$ and a reliable interaction between complexity and ISI $[F(6,66)=3.714, p<.005]$.

Array 1 Error. There was a reliable main effect of complexity $[F(2,22)=49.081$, $p<.001]$, a reliable main effect of ISI $[F(3,33)=60.369, p<.001]$ and a reliable interaction between complexity and ISI $[F(6,66)=3.170, p<.01]$. These data for accuracy and percentage of Array 1 error were illustrated in Fig. 3. 

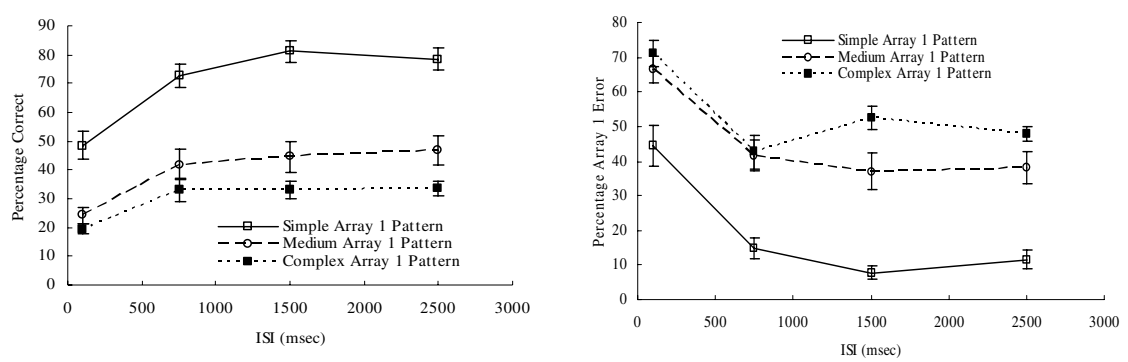

Fig. 3. Localization accuracy (the left) and array 1 error (the right) as a function of ISI and pattern complexity in present experiment. Error bars represent standard errors of the means.

Array 2 Error. Although there were a reliable main effect of complexity $[F(2,22)=$ $8.856, p<.005]$, a reliable main effect of ISI $[F(3,33)=5.095, p<.01]$ and a reliable interaction between complexity and ISI $[F(6,66)=2.310, p<.05]$, array 2 error is less than array 1 error in all conditions.

\subsection{Eye Movement Behavior}

To some extent, the locus of attention is indicated by the location in space that is fixated. Thus an analysis of modes in the distribution of fixations during the ISI separating the arrays can give insight into the manner in which attention is used during VSTM consolidation. In this section, fixation number, total fixation duration, average duration, fixation location and average pupil size were analyzed.

Fixation Number. ANOVA showed that the effects of different pattern complexities on fixation number were the same and there was a significant main effect of ISI $[F(3$, $33)=21.803, p<.001]$. The Pearson correlation between ISI and number of fixations was .99. As Fig. 4. (the left) illustrated, a linear regression analysis indicated that one additional fixation was made for every $1,000-\mathrm{msec}$ increase in ISI.
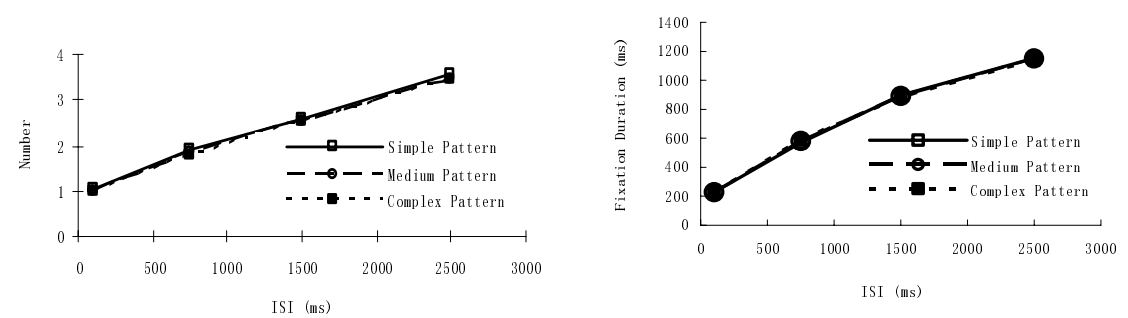

Fig. 4. The average fixation number (the left) and fixation duration (the right) in a trial in the experiment

Total Fixation Duration. Average total fixation durations on each trial were illustrated in Fig. 4. (the right) as a function of pattern complexity and ISI. An overall main effect of ISI was observed, since fixation durations generally increased with increases in ISI. ANOVA revealed that the effects of different pattern complexities on fixation 
duration were the same and there was a significant main effect of ISI $[F(3,33)=$ $36.886, p<.001]$.

Average Fixation Duration. Average fixation duration on each trial across ISIs was $666.8 \mathrm{~ms}, 684.7 \mathrm{~ms}$ and $664.7 \mathrm{~ms}$ respectively under simple, medium and complex pattern conditions. ANOVA showed that the differences of average fixation duration among three pattern complexity conditions were not significant $[F<1, p>.05]$.

Fixation Location. Fixation location under the conditions of different ISIs and pattern complexities was illustrated in Fig. 5 (the left). The data revealed that the percentage of fixations on Array 1 dots was lower than chance level (50\%) at any ISIs. ANOVA showed that there was a reliable main effect of pattern complexity $[F(3,33)=12.753$, $p<.001]$ and a reliable interaction between pattern complexity and ISI $[F(6,66)=$ $3.918, p<.005]$. Average percentage of fixation on dot locations across ISIs were $42.12 \%, 37.77 \%$, and $44.39 \%$ respectively under simple, medium and complex pattern conditions, which were all below chance level [ $t \mathrm{~s}(11) \mid>4, p \mathrm{~s}<0.01]$.

Pupil Size. Pupil size, measured by pupil diameter, under the condition of different ISIs and pattern complexities was illustrated in Fig. 5. (the right) ANOVA revealed that there was a reliable main effect of ISI $[F(3,33)=61.278, p<.001]$.
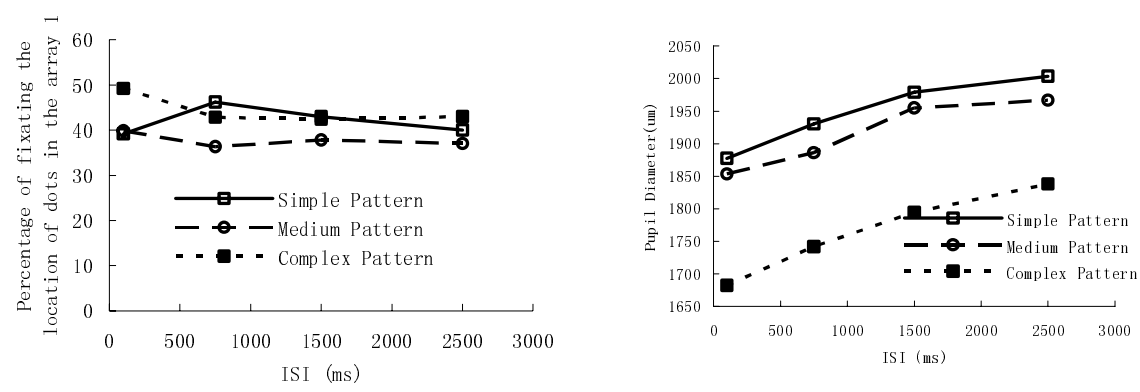

Fig. 5. The percentage of fixations on location originally occupied by a dot from Array 1 (the left) and average pupil diameter under the condition of different ISIs and pattern complexity (the right) in the experiment

\section{Discussion}

The effect stimuli complexity on information integration between VSTM and visual perception was studied in the present study. It was found that the accuracy in the simple pattern condition was higher than that in the medium pattern condition, which was higher than that in the complex condition, especially at long ISIs. Meanwhile, the array 1 error and the array 2 error also showed the similar trends. These findings supported the figural grouping hypothesis in VSTM and suggested that performance at long ISIs was supported by a limited-capacity, object-based visual memory that is sensitive to higher order image structure [10]. The effect of pattern complexity provided a means to reconcile apparently high-capacity memory at long ISIs with evidence that VSTM has a limited capacity of three or four objects. At long ISIs, 
Array 1 is likely represented in VSTM as one or more higher order objects, each containing information from more than one array element.

Average fixation numbers on each trial were positively correlated with ISI, since longer ISI afforded more time for participants to make more eye movements. This finding showed that participants had eye movements during ISI. Fixation duration had been considered to reflect the amount of processing [8] [15] [19]. In the present study, Average total fixation duration observed on each trial was positively correlated with ISI, and average fixation duration under three pattern conditons were almost the same. Altogether, eye movements seemed to be a way which helped participants to retain information of array 1 in VSTM.

More importantly, fixation location analyses showed that the percentage of fixations on locations originally occupied by dots in Array 1 was less than chance level (0.50) in three pattern conditions. This finding suggested that the participants were more likely to pay attention to empty cell locations other than locations originally occupied by dots to retain array 1 . Given that average duration of each fixation was almost the same under three pattern conditions, the total duration participants fixating locations of empty cells in array 1 was longer than total duration the participants fixating dots in array 1. These findings suggested that participants were more likely to try to retain locations of empty cells than locations of dots in array 1 during ISI. From aspect of eye movements, these results offer the first evidences supporting convert-and-compare hypothesis [13]. Brockmole et al (2005) also minitored the participants' eye movement and found that the percentage that participants fixate the grid position occupied by dots of array 1 was under random level [1]. The reasons for the difference between their results and the present study were not clear yet. One possible explanation could be the manipulation of pattern complexity in the present study. In Brockmole et al's study, the dot position in array 1 was randomly distributed, thus the dot pattern of array 1 is much similar to the complex pattern in the present study. It should be noted that the percentage of fixation on complex pattern of dots was the highest, though it was still under chance level.

Related studies [16] [20] indicated that pupil size was relevant to the mental workload of observers. Although there was a trend that average pupil size in the simple pattern and medium pattern conditions were larger than that in the complex pattern condition, the differences among the three pattern complexities were not significant. One possible explanation was that pupil size was not sensitive to the mental workload of observers, consistent with some prior study [16]. An alternative explanation was that the differences of mental workload under three pattern complexities were not strong enough.

Acknowledgement. This research was supported in part by grants from 973 Program of Chinese Ministry of Science and Technology (\#2002CB312103) and the National Natural Science Foundation of China (\#60433030 and \#30500157).

\section{References}

1. Brockmole, J.R., Irwin, D.E.: Eye movements and the integration of visual memory and visual perception. Perception \& Psychophysics 67, 495-512 (2005)

2. Brockmole, J.R., Wang, R.F.: Integrating visual images and visual percepts across time and space. Visual Cognition 10, 853-873 (2003) 
3. Brockmole, J.R., Irwin, D.E., Wang, R.F.: The locus of spatial attention during the temporal integration of visual memories and visual percepts. Psychonomic Bulletin \& Review 10, 510-515 (2003)

4. Brockmole, J.R., Wang, R.F., Irwin, D.E.: Temporal integration between visual images and visual percepts. Journal of Experimental Psychology: Human Perception \& Performance 28, 315-334 (2002)

5. Di Lollo, V.: Temporal integration in visual memory. Journal of Experimental Psychology: General 109, 75-97 (1980)

6. Di Lollo, V., Hogben, J.H.: Suppression of visible persistence as a function of spatial separation between inducing stimuli. Perception \& Psychophysics 41, 345-354 (1987)

7. Dixon, P., Di Lollo, V.: Beyond visible persistence: An alternative account of temporal integration and segregation in visual processing. Cognitive Psychology 26, 33-63 (1994)

8. Henderson, J.M., Weeks, P.A., Hollingworth, A.: The effects of semantic consistency on eye movements during complex scene viewing. Journal of Experimental Psychology: Human Perception and Performance 25, 210-228 (1999)

9. Hogben, J.H., Di Lollo, V.: Perceptual integration and perceptual segregation of brief visual stimuli. Vision Research 14, 1059-1069 (1974)

10. Hollingworth, A., Hyun, J., Zhang, W.: The role of visual short-term memory in empty cell localization. Perception \& Psychophysics 67, 1332-1344 (2005)

11. Ichikawa, S.: Quantitative and structural factors in the judgment of pattern complexity. Perception \& Psychophysics 38, 101-109 (1985)

12. Irwin, D.E.: Information integration across saccadic eye movements. Cognitive Psychology 23, 420-456 (1991)

13. Jiang, Y., Kumar, A., Vickery, T.J.: Integrating sequential arrays in visual-short term memory. Experimental Psychology 52, 39-46 (2005)

14. Jiang, Y., Olson, I.R., Chun, M.M.: Organization of visual short-term memory. Journal of Experimental Psychology: Learning, Memory, \& Cognition 26, 683-702 (2000)

15. Just, M.A., Carpenter, P.A.: A theory of reading: From eye fixations to comprehension. Psychological Review 87, 329-354 (1980)

16. Lin, Y., Zhang, W.J., Watson, L.G.: Using eye movement parameters for evaluating human-machine interface frameworks under normal control opperation and fault detection situations. International Journal of Human-Computer Studies 59, 837-873 (2003)

17. Luck, S.J., Vogel, E.: The capacity of visual working memory for features and conjunctions. Nature 309, 279-281 (1997)

18. Pashler, H.: Familiarity and visual change detection. Perception \& Psychophysics 44, 369 378 (1988)

19. Rayner, K.: Eye movements in reading and information processing: 20 years of research. Psychological Bulletin 124, 372-422 (1998)

20. Recarte, M.A., Nunes, L.M.: Mental workload while driving: effects on visual searclh, discrimination, and decision making. Journal of Experimental Psychology: Applied 9, 119-137 (2003)

21. Watson, D.G., Humphreys, G.W.: Visual marking: prioritizing selection for new objects by top-down attentional inhibition of old objects. Psychological Review 104, 90-122 (1997)

22. Watson, D.G., Humphreys, G.W.: Visual marking and visual change. Journal of Experimental Psychology: Human Perception and Performance 28, 379-395 (2002) 